\title{
Mobile Cloud Computing for Biometric Applications
}

\author{
Milos Stojmenovic \\ Department of Informatics and Computation \\ Singidunum University \\ Belgrade, Serbia \\ mstojmenovic@singidunum.ac.rs
}

\begin{abstract}
The cloud computing concept became popular in 2006 and encapsulates a business model of providing services across the Internet. The Mobile Cloud Computing (MCC) concept was proposed in 2007. Recent advances in cloud computing have given a platform to various computationally heavy tasks and made them readily accessible to even mobile devices. Biometric applications are dedicated to fingerprint, face, or iris scanning and they typically work in a laboratory setting where the client computer has unlimited access to the throughput and computational resources of the network. Mobile devices can bring biometric evidence back to the laboratory in order for it to be processed. On a cloud infrastructure, information processing could be completed much faster. The limiting factor then becomes the battery power of the device and the throughput of the communication channel of the client node to the cloud. It is important to reduce as much as possible the packet size of the query or task given to the cloud, and also minimize the size of the received response to be able to incorporate cloud biometric technology for real time evidence processing. This position paper sets the mobile cloud computing agenda for biometric applications.
\end{abstract}

Keywords-cloud biometrics, fingerprint, iris, face recognition

\section{INTRODUCTION}

Recent advances in cloud computing have given a platform to various computationally heavy tasks and made them readily accessible to even mobile devices. By transferring the load from a computationally weak mobile device to a readily accessible and powerful cloud server (composed of data center network) via mobile service network (see Figure 1), the limiting factor in the range of software possibilities of such infrastructure is the battery power of the device and the throughput of the communication channel of the client node to the cloud. Mobile devices have limits on available battery life, therefore any reduction in processor and communications use is welcome. It is important to reduce as much as possible the packet size of the query or task given to the cloud, and also minimize the size of the received response.

The MCC concept incorporates the consumer, enterprise, femtocells, transcoding, end to end security, home gateways, mobile broadband enabled services etc. With 'dumb' terminals and an intelligent network, a customer can 'put' the cloud in his or her pocket. RIM's blackberry is an early product following MCC, with employees getting access to company's emails, databases, and instant message servers. Apple's iCloud is a solution based on cloud storage and cloud computing, handling email, notepad items, contacts book, photos and documents, automatically synchronized to iMac, iPod, iPhone and other Apple's terminal devices. Microsoft's "LiveMesh" integrates Windows computers, Windows Mobile cell phones, Xbox, even Mac computers and cell phones running other operating systems with open interface, interconnects these devices through Internet, thus enables users to synchronize between different devices and the Internet, and store their data in the "cloud". MCC technologies are emerging, and academia is now becoming increasingly active, with several newly established workshops devoted exclusively to this topic (e.g., Mobicc at IEEE ICCC 2012).

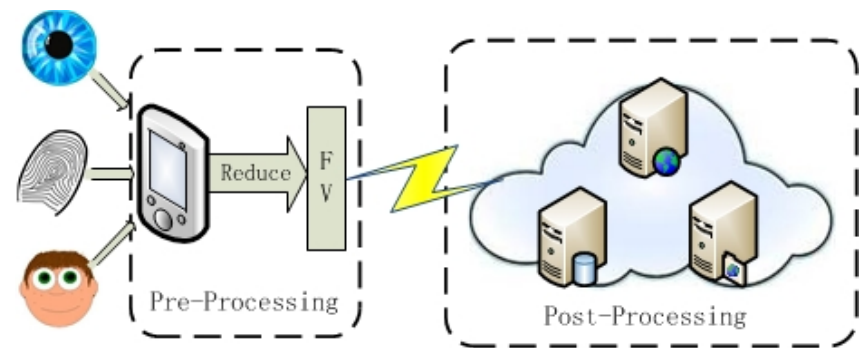

Figure 1. Example of Mobile extracts feature vector and offloads recognition task to the cloud

We are concerned with energy consumption at the user side, in addition to computing and storage limitations. It consists of transmission (network interface), computation (CPU, memory), and sensing (camera, GPS) costs. References addressing these costs can be found in [CMWSL]. Transmission challenges include unstable wireless quality, heterogeneous interfaces, and different traffic demands for real-time and delay-tolerant applications. Solutions include sleeping during idle time, predicting signal strength \& traffic pattern to avoid rush hour, and sending in a burst by traffic shaping. Computation optimizations are achieved mainly by task out-sourcing to the cloud and CPU optimization. High computation and low transfer tasks are ideal candidates for outsourcing. Some tasks are always outsourced, some never, and for some it depends on bandwidth and other factors.

Power modeling and energy state prediction can produce satisfactory decisions. Challenges on energy consumption for sensing include high-energy consumption 
of specific sensors (in particular, GPS for location-based service), static use of location sensing mechanisms, and lack of cooperation among multiple location-based applications (LBAs). Solutions include substitution (making use of alternative location-sensing mechanism), suppressing unnecessary GPS sensing (assisted by less power-intensive sensors to detect state e.g., accelerometer), and piggybacking (synchronizing the location sensing requests from multiple running LBAs).

Biometric applications are mainly based on face, fingerprint and iris detection, verification and identification systems. Detection systems only recognize the existence of a face, fingerprint or iris in an image, but do not match it with any individual. It is normally the first step in computer vision tasks, discovering the regions of interests in the image. Verification systems usually output a decision on whether a query matches the holding template on a one-toone basis, and commercial products have been available on the market. We are primarily interested here in the identification systems which are much more challenging, which suggest whether or not query can find a match in the database, possibly giving several options with their likelihood (a one-to-many matching process).

Software dedicated to fingerprint, face, iris or any other biometric scanning which identifies and verifies individuals typically works in a laboratory setting where the client computer has unlimited access to the throughput and computational resources of the network. The limitation of this architecture is typically physical, which means that a user or client terminal needs to be in the resident building of the infrastructure for the operation to work smoothly. A minimal requirement is to be linked to the network, and be able to pass significant quantities of data between the client and the mainframe computers. For this reason, police bring biometric evidence back to the laboratory in order for it to be processed. Using a cloud infrastructure, information processing could be completed much faster provided that the sizes of the queries and resulting data sets can be reduced to minimize bandwidth costs.

\section{BACKGROUND AND STATE OF THE ART}

Over the last few years, research has focused on applying cloud computing to mobile devices, varying from new computing offloading system structures, task partitioning schemes, virtual machine based process/runtime migration, to reducing server response latency by using intelligent surrogate discovery protocol, and scheduling specific protocol-level energy saving schemes (e.g. WiFi PowerSaving Mode (PSM) or cellular Radio Resource Control (RRC)) to lower wireless communication energy. Recent surveys of 'green' wireless transmission and modeling and location based applications in MCC are given in [CMWSL] and [MCS], respectively. Data redundancy elimination in data centers with wireless cards and routers was studied in [CLSLW], which deals with cloud server issues.

\section{A. MCC technologies}

The key idea in [GRJKA] is to model the dependencies between application modules, and optimize the partitions of MCCs. The key problem is to partition an algorithm between the mobile and the server (the cloud), considering also their dependency. Hyrax, making use of MapReduce, Hadoop, and Android, is an infrastructure that enables smartphone applications with distributed data and computation (http://www.stormingmedia.us/10/1062/A106215.html), to tackle the limitation of storage and computing ability. Cloudlet [SBCD] is the third layer (part of fixed infrastructure) device, ensuring low end-to-end latency, e.g., for body-worn computer in cognitive real time assistance (computer vision, language) applications. It acts as a 'data center in a box' in places (e.g., coffee shops) with high speed Internet access.

\section{B. Applications}

The smartphone is the primary mobile device for MCC applications, followed by the laptop, embedded board and robot [CMWSL]. Cloud robotics is an emerging application area, with several ongoing projects, e.g., a cloud infrastructure to perform speech recognition, face detection, and other tasks that might help improve robot interaction with patients [G]. Existing research articles describe multimedia processing, image/vision, graphics, gaming and text processing applications. Our primary interests are in image processing applications, focusing on biometric ones. Consider two motivating examples. A photo could be taken of a fellow pedestrian, with a miniature camera located in eye glasses. The image can be pre-processed to extract features (compressed but sufficient information about the face) and wirelessly sent to a database server for identification, and the name of the person could be returned to the glasses. Similar ideas are being implemented by the sixth sense project at MIT [M] and Google's project Glass [PLT]. A video camera could monitor e.g., entrance to a bank, and send movement extracted features for detecting unusual behavior. If a zoomed image could identify the detected face, the robber could be identified in real time.

\section{Fingerprint recognition}

Fingerprint evidence in forensic sciences has been an integral tool in the criminal justice system for over a century in many nations. Recently, fingerprint evidence has been disputed in several criminal cases, and some wrongful accusations were revealed. Linking partial fingerprint to a unique source is sustained largely by faulty probabilistic intuition that equates infrequency with uniqueness [SK]. Nevertheless errors are rare and it remains widely accepted single most valuable tool in forensic evidence. Interpol introduced an automated fingerprint matching system in 2005 with a database consisting of 50,000 sets of fingerprints, and the US department of Homeland Security introduced a database consisting of 120 million sets of fingerprints in 2012. Each country compiles its own database of fingerprints. Software for accurate fingerprint matching is still an active research topic, and an essential one for large 
datasets where manual search by fingerprint experts is infeasible.

Fingerprint verification based systems are easier to build than identification ones. In [RRCG], a mobile biometric system for signing digital transactions is described. In [WHP], a fingerprint orientation model based on 2D Fourier expansions (FOMFE) in the phase plane was proposed. It does not require prior knowledge of singular points, and is able to describe ridge topology seamlessly, including singular points, even for noisy fingerprints. Instead of row orientation field data (gradients of image intensity changes), it uses FOMFE coefficients directly as the indexing feature. The resulting feature vector, after parameter tuning and for selected fingerprint databases, is of length 162, about ten times smaller. The pre-processing time (to compute the feature vector) and post-processing times (reconstruction) are linear in the number of points from the coarse input. Similarity (and matching) is based on the Euclidean distance in the feature space, with the previously reduced dimensionality via a suitable standard transform.

A fingerprint recognition system for cloud computing communications was described in $[\mathrm{Y}+]$. With the benefit of the Fourier Transform image enhancement, the reference point can be reliably and accurately determined by the complex filtering methods. The features extracted by using the geometry moment and Zernike moment analysis on subROIs have covered both local and global properties. Matching the fingerprints is implemented by the Support Vector Machine (SVM) classifier. Although the feature vector is only of length 68, its computation involves complex Zernike moments, and is not suitable for smart phones.

\section{Face detection and recognition}

Face detection has been one of the most studied topics in the computer vision literature. [ZZ] surveyed recent advances in face detection for the past decade (extending a highly cited survey [HAJ]). The seminal Viola-Jones face detector $[\mathrm{VJ}]$ is featured. Various techniques are classified according to how and what kind of features they extract, and what learning algorithms are adopted. Our team tested the algorithm [ZLZW] which is a better algorithm than [VJ] due to its rotation invariance. [AB] proposed a MCC extensible system architecture for context-aware navigation by exploiting the computational power of resources made available by cloud computing providers as well as the wealth of location-specific resources available on the Internet. It includes face recognizer in the cloud with input coming from camera module integrated into sunglasses. Their face recognizer is based on Viola-Jones AdaBoost features [VJ]. This algorithm detects the presence of a face in real time (which helps in social interactions), but does not identify a particular face.

Face recognition is based on face detection as the first step, followed by extracting suitable features, which are then used in database matching for verification and identification. Various techniques for still and video-based face recognition are surveyed in [ZCPR] (a more recent survey is given in
[ANRS]). Finding local discriminate facial features that can be used to determine identity has been a research topic since 1995. The Face Recognition Grand Challenge was a competition initiated and held by the US government from 2004 - 2006 in order to advance the field of face recognition, and it was determined that algorithms at the end of the competition were 100 times more accurate than those in 1995, and some were able to outperform human participants in recognizing faces and could uniquely identify identical twins [W].

However, these tests represent laboratory experiments where faces were scanned in high resolution, under ideal lighting conditions. In practice, existing systems are notoriously inefficient and inaccurate, as experienced by various law enforcement agencies in numerous countries. For example, one district in London has employed a face recognition system on street cameras which has reportedly never been able to identify a single criminal, despite being active for several years. Similar failures were noted in Tampa, Florida, and Logan International Airport in Boston, respectively, where face recognition systems failed to make a single match in several years [14]. Facial expression can radically effect the outcome of a successful match even under ideal lighting conditions, which is why Canadian passport photos are now taken while the subject is expressionless.

There have been several attempts to integrate the facial recognition capabilities to mobile devices. Sony Ericsson's "Recognizer" application on their X10 hardware enables the user to take a picture using the camera and recognize people present in the picture. It stores the pictures locally in the device and only recognizes people who are present in the contacts list and have a picture associated. Cloud technology and social networks are not used. Viewdle's mobile application allows detecting people from one picture taken with the camera with contacts from a Facebook account. It runs the recognition process entirely in the mobile device. [SPF] built an Android application, CroudSTag, which aids in forming social groups of common interest, from the mobile devices. The application obtains a set of pictures from a storage cloud, uses the face recognition cloud services (face.com) to identify the people, and forms social groups on Facebook. However the reported average response time is 22 seconds, which is slow. [TP] described a highly cited approach to the near-real-time detection and identification of human faces. Face images are projected onto a feature 'eigenfaces', which are the eigenvectors of the set of faces. In [CTCH], a fast access control applied to Hadoop cloud computing using the fingerprint identification and face recognition is introduced. The paper did not state which of the underlying algorithms was applied.

\section{E. Iris recognition}

The most popular iris recognition approach was proposed by Daugman [D], where the pupil and outer iris boundary are first detected, the iris 'ring' is extracted and the code is obtained from it, and then matched in the database. All iris recognition systems undergo the same steps when 
performing identity validation. Daugman [D] proposed an exhaustive search over the entire image in order to locate the inner and outer iris rings, taking into account 3 parameters $(X 0, Y O, R)$, where $X 0$ and $Y 0$ are the proposed coordinates of the center of the eye, and $R$ is the radius from the center to the boundary. Each iris has 2 radii (one to the edge of the pupil, and the other to the edge of the iris). This method extracts keys using 2D Gabor wavelets over gray scale images of the iris. The recognition principle is the failure of a test of statistical independence on iris phase structure encoded by multi-scale quadrature wavelets. The initial exhaustive search step is the computational choke point of this algorithm. It produces an accurate location of the iris, at the cost of an $\mathrm{O}\left(n^{2} \log n\right)$ algorithm for an $n \times n$ image. In [KSTM], a prototype system for matching a collection of synthetic human iris images, based on [D], is described. It is implemented in a cloud computing (not a MCC) environment. The size of feature vector (iris code) was not discussed in [D, KSTM]; however the search space has about 250 degrees of freedom in [D] which could serve as a feature vector, which might be somewhat large for MCC. A less demanding algorithm needs to be developed in order to be included on mobile devices.

\section{RESEARCH AGENDA}

Biometric applications on MCC would directly benefit law enforcement agencies in processing forensic requests directly from the crime scene or enable biometric security inspection remotely via battery powered devices. It would reduce the cost of biometric security devices since they wouldn't have to be physically connected to the network in order to function. The intended real time performance of face recognition scheme also leads toward novel applications such as instant name recognition with camera placed in eye glasses. The technical content of each biometric application is described next.

\section{A. Fingerprint identification}

The recent algorithm in [YHS] reduces the size of query feature vector (to be transmitted by mobile), compared to the state of the art, with a simpler and intuitively clearer strategy, while preserving its matching power. It is based on extracting minutiae from the fingerprint, and constructing Delaunay triangulation from it, and measuring similarity of the corresponding two planar graph. The matching algorithm may return several best matches, each with their matching likelihood measure.

Although fast derivation of the feature vector of short length 162 in [WHS] appears reasonable for performing on a mobile and transferring to a cloud server, we observe that a different and simpler concepts may give even better starting point. A possible solution to the problem to query size reduction is to reformat the input image into a planar graph like representation that is easily stored, sent and most importantly matched against candidate indicators. [YHS] proposed to first compute minutiae points from fingerprints.
Minutiae represent the internal endpoints of lines and can be extracted by computer software such as Verifinger.

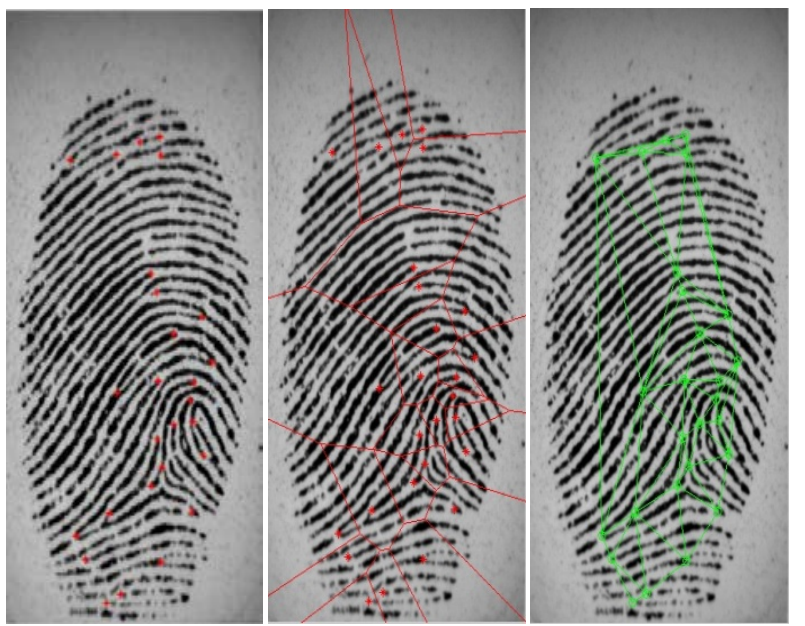

Figure 2. Extraction of feature vector from minutia corners, their Voronoi diagram, and Delaunay triangulation

In the example in Fig. 2, less than 30 minutia are extracted. The feature vector of extracted minutia generally consists of the minutia type, the coordinates and the tangential angle of the minutia. If only point coordinates are used, feature vectors of length less than 60 can be defined for transmission from the mobile device to the server. A planar graph out of these points can be constructed by first computing a Voronoi diagram of the point set, and then joining adjacent regions. Figure 2 offers a description of the basic steps. The challenge is to design a planar graph matching algorithm that will compare the corresponding structures of a query fingerprint and those in the database.

\section{B. Face recognition}

Although some MCC face recognition solutions exist, they lack real time performance. The name of the person seen on the street should be revealed on the glasses (with augmented reality) in real time to be socially effective. Among the plethora of existing solutions to this well studied problem, it would be a daunting task to identify those that provide a basis for real time recognition. The most likely winning approach would be on identifying key feature points on the face, and studying their structure toward defining a novel similarity measure.

A similarity measure for facial recognition could be based on the relative distances between key facial features or key facial ratios, such as the distance between the eyes, the width of the face, the horizontal and vertical measurements of the mouth, the ratio of the width between the eyes to the width of the largest part of the nose, etc. Recently, it has been shown that including the texture of the skin in the map of the face can ameliorate the matching performance by as much as $20-25 \%$ [W]. This means that individual marks on the face such as scars or moles can significantly increase accuracy if incorporated into existing 
designs. A graph of feature points is used as the ordered feature vector for facial recognition (see Figure 3).
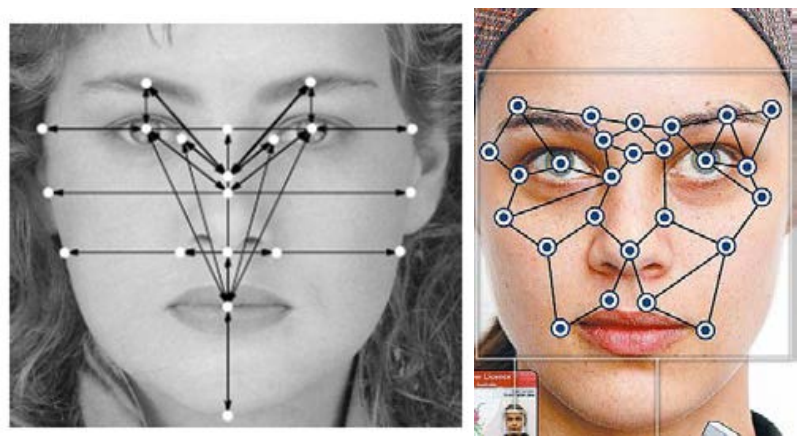

Figure 3. Examples of key facial feature points

\section{Iris recognition}

Iris detection can be simplified by applying the method from [SN] which detects circles by finding the true centers of detected lines, their polar mapping, and measuring their linearity. The method addresses circle imperfections by requiring only partial circle around the true center. For iris recognition, it is not necessary to use all Gabor wavelet coefficients from [D]. It is well known that most of coefficients at the tail are irrelevant for the reconstruction process. Therefore a reasonable number of low indexed coefficients is expected to provide satisfactory outcome, while serving as small feature vector.

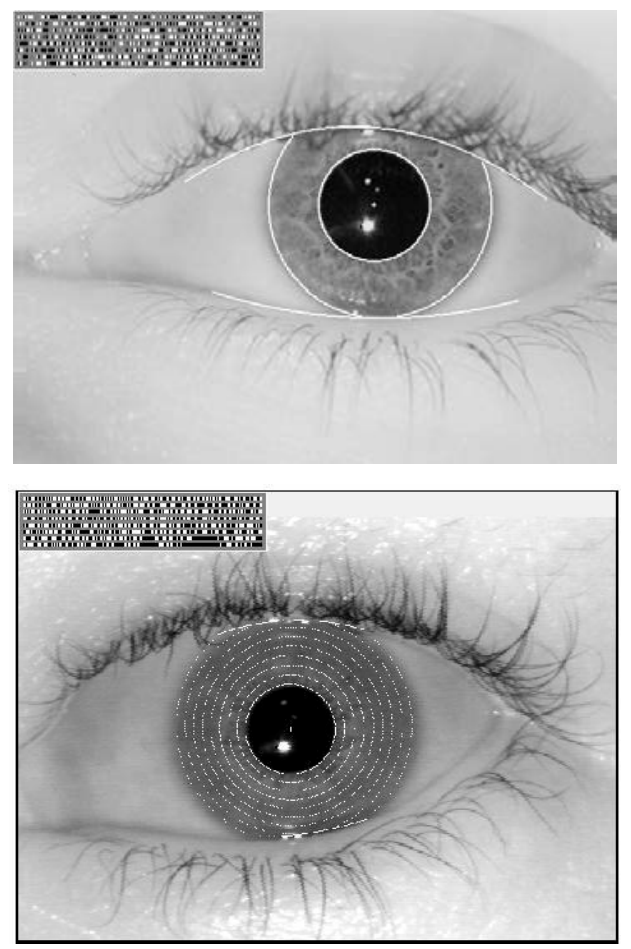

Figure 4. Iris code detection and extraction

State of the art algorithms for identification based on iris recognition use infra red emitters and cameras to more easily locate the iris. This poses an additional hardware requirement, but not an expensive one. The iris area is located using various circularity detection algorithms, and the iris codes are extracted using an array of adjacent circular rings starting at the pupil, and ending at the sclera (see Figure 4 for clarification). Iris data that is extracted from grayscale images is discriminant enough to generate reliable 128 bit codes that can identify people from a large database.

The experimental setup could consist of the network of computers as well as one or two mobile phones running on the open source android platform [A]. A virtual private network can be formed between the mobile devices and the network of computers, or the computers can act as servers and listen to web requests issued by the mobile devices via their Wifi Internet connections. The simulations could measure the energy consumption of the devices, the lag between issued queries and retrieved results, bandwidth consumed, and accuracy of the results. The goal of the simulation is to develop an application for the android platform that takes a snapshot of a biometric indicator (fingerprint, face, iris) using its built in camera of adequate resolution, reduces the size of this indicator to a few bytes and sends the reduced representation to the servers. It then waits for a response and displays the data to the user.

\section{ACKNOWLEDMENT}

This work was partially supported by the following grant: "Digital signal processing, and the synthesis of an information security system", TR32054, Serbian Ministry of Science and Education.

\section{REFERENCES}

[A] Open Source Android Platform, http://source.android.com/, 2012.

[AB] P. Angin, B. K. Bhargava, Real-time Mobile-Cloud Computing for Context-Aware Blind Navigation, International Journal of Next-Generation Computing, Vol. 2, No. 2, July 2011.

[ANRS] A. F. Abate, M. Nappi, D. Riccio, G. Sabatino, 2D and 3D face recognition: A survey, Pattern Recognition Letters, Volume 28, Issue 14, 15 October 2007, 1885-1906.

[CTCH] B.R. Chang, H.F. Tsai, C.M. Chen, C.F. Huang, Access Control of Cloud Computing Using Rapid Face and Fingerprint Identification, $2^{\text {nd }}$ Int. Conf. Innovations in Bio-inspired Computing and Applications (IBICA), 179-182, Dec. 2011.

[CMWSL] Y. Cui, X. Ma, H. Wang, I. Stojmenovic, J. Liu, A Survey of Energy Efficient Wireless Transmission and Modeling in Mobile Cloud Computing, Mobile Networks and Applications MONET, to appear.

[CLSLW] Y. Cui, C. Liao, I. Stojmenovic, Di Li, H. Wang, Cooperative Redundancy Elimination in Data Center Networks with wireless cards at routers, 2nd International Workshop on Data Center Performance, DCPerf-2012, at 32nd IEEE ICDCS, Macau, June 21, 2012, to appear.

[D] J. Daugman, How iris recognition works, IEEE Transactions on Circuits and Systems for Video Technology, Vol. 14, No. 1, January, 2004.

[G] E. Guizzo, Robots with their heads in the clouds, IEEE Spectrum, vol. 48, no. 3, 16-18, March 2011. 
[GRJKA] I. Giurgiu, O. Riva, D. Juric, I. Krivulev, and G. Alonso, Calling the cloud: enabling mobile phones as interfaces to cloud applications, ACM 10th Int. Conf. Middleware, Springer, 83102, 2009.

[HAJ] R.L. Hsu, M. Abdel-Mottaleb, A.K. Jain, Face detection in color images, IEEE Transactions on Pattern Analysis and Machine Intelligence, vol. 24, no. 5, 696-706, May 2002.

[KSTM] E. Kohlwey, A. Sussman, J. Trost, A. Maurer, Leveraging the Cloud for Big Data Biometrics: Meeting the Performance Requirements of the Next Generation Biometric Systems, IEEE World Congress on Services (SERVICES), 597-601, July 2011.

[L] S.Z. Li (ed.), Encyclopedia of Biometrics (1st ed.), Springer 2009.

[M] P. Mistry, SixthSense: A Wearable, Gestural Interface to Augment Our World, http://www.media.mit.edu/research/highlights/sixthsensewearable-gestural-interface-augment-our-world, MIT, 2009.

[MCS] X. Ma, Y. Cui, I. Stojmenovic, Energy Efficiency on Location Based Applications in Mobile Cloud Computing: A Survey, The 9th International Conference on Mobile Web Information Systems (MobiWIS), Niagara Falls, August 27-29, 2012; Procedia Computer Science, to appear.

[PL] B. Parviz, S. Lee, S. Thrun, Project Glass, plus.google.com/ 111626127367496192147/posts, 2012.

[RRCG] R. Ribalda, G.G. de Rivera, A. de Castro, J. Garrido, A Mobile Biometric System-on-Token System for Signing Digital Transactions, IEEE Security \& Privacy, , vol. 8, no. 2, 13-19, March-April 2010.

[SBCD] M. Satyanarayanan, P. Bahl, R. Caceres, N. Davies, The Case for VM-Based Cloudlets in Mobile Computing, IEEE Pervasive Computing, vol. 8, no. 4, 14-23, Oct.-Dec. 2009.

[SK] M. Saks, J. Koehler, The Individualization Fallacy in Forensic Science Evidence, 2007.

[SN] M. Stojmenovic, A. Nayak, Shape based circularity measures of planar point sets, IEEE Int. Conf. on Signal Processing and Communications, 1279-1282, Dubai, UAE, 24-27 Nov 2007.

[SPF] S. N. Srirama, C. Paniagua, H. Flores, CroudSTag: Social Group Formation with Facial Recognition and Mobile Cloud Services, Procedia Computer Science, 5, 2011, 633-640.
[TP] M.A. Turk, A.P. Pentland, Face recognition using eigenfaces, IEEE Computer Vision and Pattern Recognition CVPR, 586591, Jun 1991.

[VJ] P.A. Viola, M. Jones, Robust real-time face detection, Int. J. Computer Vision, 57, 2, 137-154, 2004.

[W] M. Williams, Better Face-Recognition Software, http://www.technologyreview.com/Infotech/18796/?a=f, $\quad$ MIT Technology Review, May 2007.

[WHP] Yi Wang, J. Hu, D. Phillips, A Fingerprint Orientation Model Based on 2D Fourier Expansion (FOMFE) and Its Application to Singular-Point Detection and Fingerprint Indexing, IEEE Transactions on Pattern Analysis and Machine Intelligence, vol. 29, no. 4, 573-585, April 2007.

[YHS] W. Yang, J. Hu M. Stojmenovic NDTC: A Novel Topologybased Fingerprint Matching Algorithm using N-layer Delaunay Triangulation Net Check, The $7^{\text {th }}$ IEEE Conference on Industrial Electronics and Applications ICIEA 2012,Singapore, July 18 20, 2012, to appear.

[Y+] J. Yang, N. Xiong, A.V. Vasilakos, Z. Fang, D. Park, X. Xu, S. Yoon, S. Xie, Y. Yang, A Fingerprint Recognition Scheme Based on Assembling Invariant Moments for Cloud Computing Communications, IEEE Systems Journal, vol. 5, no. 4, 574-583, Dec. 2011.

[ZCPR] W. Zhao, R. Chellappa, P. J. Phillips, and A. Rosenfeld, Face recognition: A literature survey. ACM Comput. Surv. 35, 4 (December 2003), 399-458.

[ZLZW] J. Zhou, X.G. Lu, D. Zhang, C. Wu, Orientation analysis for rotated human face detection, Image and Vision Computing, 20, 2002, 257-264.

[ZZ] C. Zhang and Z. Zhang, A Survey of Recent Advances in Face Detection, Technical Report MSR-TR-2010-66, Microsoft Research, June 2010. 